\title{
Upper bound on the gravitational masses of stable spatially regular charged compact objects
}

\author{
Shahar Hod \\ The Ruppin Academic Center, Emeq Hefer 40250, Israel \\ and \\ The Hadassah Institute, Jerusalem 91010, Israel
}

(Dated: March 27, 2019)

\begin{abstract}
In a very interesting paper, Andréasson has recently proved that the gravitational mass of a spherically symmetric compact object of radius $R$ and electric charge $Q$ is bounded from above by the relation $\sqrt{M} \leq \frac{\sqrt{R}}{3}+\sqrt{\frac{R}{9}+\frac{Q^{2}}{3 R}}$. In the present paper we prove that, in the dimensionless regime $Q / M<\sqrt{9 / 8}$, a stronger upper bound can be derived on the masses of physically realistic (stable) self-gravitating horizonless compact objects: $M<\frac{R}{3}+\frac{2 Q^{2}}{3 R}$.
\end{abstract}

\section{INTRODUCTION}

The asymptotically measured gravitational mass $M$ of a spherically symmetric asymptotically flat Schwarzschild black-hole spacetime is directly related to the horizon radius $R$ by the simple relation $M=R / 2$ [1, 2]. It is well known that a stronger upper bound on the gravitational masses of spatially regular self-gravitating horizonless compact objects is provided by the physically important Buchdahl bound $M \leq 4 R / 9[3]$.

Similar bounds are known to exist for charged self-gravitating compact objects. In particular, charged ReissnerNordström black holes are characterized by the simple relation $M=R / 2+Q^{2} / 2 R$ [1]. In a physically interesting paper, Andréasson [4] has recently derived the stronger upper bound

$$
\sqrt{M} \leq \frac{\sqrt{R}}{3}+\sqrt{\frac{R}{9}+\frac{Q^{2}}{3 R}}
$$

on the gravitational masses of spatially regular horizonless charged compact objects.

In the present paper we raise the following physically intriguing question: Can one improve the important upper bound (11) on the masses of self-gravitating charged compact objects by adding to the characteristic properties of the corresponding horizonless curved spacetimes the physically motivated requirement of dynamical stability?

As we shall explicitly show below, the above stated question is directly related to the physically important theorem presented recently in [5] (see also [6, 7]), according to which the innermost null circular geodesic of an horizonless compact object, if it exists, is stable [8]. In particular, combining this interesting physical property of the spatially regular self-gravitating compact objects that we consider in the present paper with the intriguing assertion made in [9] (see also [10, 11]), according to which horizonless spacetimes which possess stable null circular geodesics (stable closed light rings) are expected to develop non-linear instabilities in response to the presence of time-dependent massless perturbation fields [12], one concludes that spatially regular compact objects that possess light rings in their exterior spacetime regions are dynamically unstable.

Motivated by the physically important observations made in [5, 9] regarding the (in)stability properties of horizonless compact objects, in the present paper we shall use analytical techniques in order to derive an improved upper bound [see Eq. (22) below] on the maximally allowed gravitational masses $M^{\max }(R, Q)$ of dynamically stable spatially regular charged compact objects.

\section{DESCRIPTION OF THE SYSTEM}

We consider self-gravitating horizonless charged compact objects whose spatially regular curved spacetimes are described by the spherically symmetric line element [1, 10, 11, 13 15]

$$
d s^{2}=-e^{-2 \delta} \mu d t^{2}+\mu^{-1} d r^{2}+r^{2}\left(d \theta^{2}+\sin ^{2} \theta d \phi^{2}\right) .
$$

The radially dependent metric functions $\mu=\mu(r)$ and $\delta=\delta(r)$ are related to the composed energy-momentum tensor $T_{\nu}^{\mu}($ total $)=T_{\nu}^{\mu}$ (matter) $+T_{\nu}^{\mu}$ (electromagnetic-field) of the charged matter configurations by the Einstein field equations [1]

$$
G_{\nu}^{\mu}=8 \pi\left[T_{\nu}^{\mu}(\text { matter })+T_{\nu}^{\mu}(\text { electromagnetic-field })\right],
$$


which, using the curved line element (2) and the functional expressions [16]

$$
T_{t}^{\mathrm{em} t}=T_{r}^{\mathrm{em} r}=-T_{\theta}^{\mathrm{em} \theta}=-T_{\phi}^{\mathrm{em} \phi}=-\frac{Q^{2}(r)}{8 \pi r^{4}}
$$

for the components of the electromagnetic (em) energy-momentum tensor, can be expressed in the differential forms [11, 16, 17]

$$
\mu^{\prime}=-8 \pi r\left[\rho+\frac{Q^{2}(r)}{8 \pi r^{4}}\right]+\frac{1-\mu}{r}
$$

and

$$
\delta^{\prime}=-\frac{4 \pi r(\rho+p)}{\mu} .
$$

Here $Q(r)$ is the electric charge contained within a sphere of areal radius $r[16], \rho \equiv-T_{t}^{t}($ matter $)$, and $p \equiv T_{r}^{r}($ matter $)$ [18].

The metric functions $\{\mu, \delta\}$ of the horizonless spatially regular asymptotically flat spacetime (2) are respectively characterized by the near-origin and far-region functional relations [11]

$$
\mu(r \rightarrow 0) \rightarrow 1 \quad ; \quad \mu(r \rightarrow \infty) \rightarrow 1
$$

and 11$]$

$$
\delta(0)<\infty \quad ; \quad \delta(r \rightarrow \infty) \rightarrow 0
$$

In particular, the Einstein equation (5) implies that the radial metric function $\mu(r)$ can be expressed in the mathematically compact form [4]

$$
\mu(r)=1-\frac{2 m(r)}{r}+\frac{Q^{2}(r)}{r^{2}}
$$

where $m(r)$ is the gravitational mass contained within a sphere of radius $r$ [4, 16]. For later purposes we note that, as explicitly proved in [11], regular self-gravitating matter configurations with asymptotically measured finite ADM masses are characterized by the asymptotically decaying functional behavior

$$
r^{3} T_{r}^{r}(\text { total }) \rightarrow 0 \quad \text { for } \quad r \rightarrow \infty
$$

\section{THE UPPER BOUND ON THE GRAVITATIONAL MASSES OF STABLE SPATIALLY REGULAR HORIZONLESS CHARGED COMPACT OBJECTS}

In the present section we shall prove that, in the dimensionless regime [19, 20]

$$
\frac{Q}{M} \leq \sqrt{\frac{9}{8}}
$$

one can use the instability properties of spatially regular horizonless spacetimes which possess light rings [5-7, 9], in order to derive an upper bound on the gravitational masses of physically realistic (stable) charged compact objects. In particular, below we shall explicitly show that the newly derived bound [see Eq. (22) below] is stronger than the important upper bound (11).

The functional equation which determines the radii of light rings in the curved spacetime (2) was derived in [1, 10, 11]. For completeness of the presentation, we shall first provide a brief sketch of the analytical derivation of the functional relation which characterizes the null circular geodesics of the charged spacetime. As explicitly shown in [1, 10], the circular null trajectories which characterize the spherically symmetric spacetime (2) are determined by the two relations [21, 22]

$$
V_{r}=E^{2} \quad \text { and } \quad V_{r}^{\prime}=0
$$

where the effective potential $V_{r}$ is given by the functional expression [1, 10, 11]

$$
E^{2}-V_{r} \equiv \dot{r}^{2}=\mu\left(\frac{E^{2}}{e^{-2 \delta} \mu}-\frac{L^{2}}{r^{2}}\right)
$$


Here the energy $E$ and the angular momentum $L$ are conserved quantities which reflect the fact that the metric components of the spherically symmetric static spacetime (2) are independent of the time and angular coordinates $\{t, \phi\}[1,10,11]$.

Substituting Eq. (13) into Eq. (12) and using the Einstein differential equations (5) and (6), one finds that the light rings of the spherically symmetric static curved spacetime (2) are determined by the compact functional relation

$$
\mathcal{R}(r) \equiv 3 \mu-1-8 \pi r^{2}\left[p-\frac{Q^{2}(r)}{8 \pi r^{4}}\right]=0 \quad \text { for } \quad r=r_{\gamma} .
$$

In addition, taking cognizance of Eqs. (7), (10), and (14), one deduces that the dimensionless function $\mathcal{R}(r)$, whose zeroes determine the discrete radii of the null circular geodesics of the spherically symmetric spacetime (2), is characterized by the two boundary relations

$$
\mathcal{R}(r=0)=2 \quad \text { and } \quad \mathcal{R}(r \rightarrow \infty) \rightarrow 2 .
$$

These simple relations imply that spatially regular horizonless compact objects are generally characterized by an even number of null circular geodesics [5, [6, 23].

The stability properties of the null circular geodesics are generally determined by the second spatial derivative of the effective curvature potential (13) [1, 10]. In particular, unstable light rings are characterized by locally concave radial potentials with $V_{r}^{\prime \prime}\left(r=r_{\gamma}\right)<0$, whereas stable circular geodesics which, as discussed in [9], are associated with non-linear instabilities of the corresponding curved spacetimes, are characterized by locally convex curvature potentials with the property $V_{r}^{\prime \prime}\left(r=r_{\gamma}\right)>0$ [1, 10]. Taking cognizance of Eqs. (15), (6), (12), and (13), and using the conservation equation $T_{r ; \mu}^{\mu}=0$ [16], one finds the simple functional relation [6, 7]

$$
V_{r}^{\prime \prime}\left(r=r_{\gamma}\right)=-\frac{E^{2} e^{2 \delta}}{\mu r_{\gamma}} \times \mathcal{R}^{\prime}\left(r=r_{\gamma}\right) .
$$

From Eqs. (14) and (15) one deduces that the innermost null circular geodesic, $r=r_{\gamma}^{\text {innermost }}$, of a spatially regular horizonless compact object is generally [23] characterized by the properties

$$
\mathcal{R}\left(r=r_{\gamma}^{\text {innermost }}\right)=0 \quad \text { and } \quad \mathcal{R}^{\prime}\left(r=r_{\gamma}^{\text {innermost }}\right)<0 .
$$

In particular, the innermost light ring of a spatially regular compact object, if it exists, is generally stable with the property $V_{r}^{\prime \prime}\left(r=r_{\gamma}^{\text {innermost }}\right)>0$ [see Eqs. (16) and (17)] [5]

The exterior spacetime regions $(r \geq R)$ of the spherically symmetric charged compact objects that we consider in the present paper are characterized by the relations [4]

$$
\rho=p=0
$$

and [1]

$$
\mu(r)=1-\frac{2 M}{r}+\frac{Q^{2}}{r^{2}} \quad \text { for } \quad r \geq R,
$$

where $\{M, Q\}$ are respectively the total gravitational mass and the total electric charge of the spherically symmetric spacetime as measured by asymptotic observers.

Let us assume that the spatially regular charged compact object possesses an external light ring with $r_{\gamma}>R$. Substituting Eqs. (18) and (19) into the functional relation (14), which characterizes the null circular geodesics of the spherically symmetric spacetime (2), one finds the remarkably simple expression

$$
r_{\gamma}^{\text {outer }}=\frac{1}{2}\left(3 M+\sqrt{9 M^{2}-8 Q^{2}}\right) \quad \text { for } \quad \frac{Q}{M} \leq \sqrt{\frac{9}{8}}
$$

for the radius of the outer light ring.

As discussed above, the presence of the light ring (20) outside the surface of a spatially regular horizonless compact object 24] implies the existence of a second (stable) light ring (with the property $r_{\gamma}^{\text {innermost }}<r_{\gamma}^{\text {outer }}$ ) in the charged curved spacetime. In particular, as suggested in [9], the presence of this inner stable null circular geodesic in the spherically symmetric curved spacetime (2) may indicate that the corresponding horizonless compact object is nonlinearly unstable to massless perturbation fields [12, 25]. One therefore concludes that spatially regular horizonless spacetimes describing physically realistic (stable) compact objects must not possess light rings. This physical fact yields the lower bound [see Eq. (20)]

$$
R>\frac{1}{2}\left(3 M+\sqrt{9 M^{2}-8 Q^{2}}\right)
$$

on the radii of stable horizonless charged compact objects. 


\section{SUMMARY AND DISCUSSION}

In a physically important paper, Andréasson [4] has recently derived the upper bound (1) on the gravitational masses of spatially regular horizonless charged compact objects. In the present paper we have raised the physically interesting related question: Can one derive a stronger upper bound on the gravitational masses of stable charged compact systems?

This physically intriguing question is motivated by the recent theorem presented in [5] which, when combined with the results presented in [9], asserts that horizonless compact objects whose curved spacetimes possess null circular geodesics (light rings) are non-linearly unstable to massless perturbation fields.

Using analytical techniques, we have proved that the answer to the above stated question is 'Yes!'. In particular, it has been explicitly proved that the masses of physically realistic (stable) self-gravitating horizonless compact objects are bounded from above by the compact functional relation [see Eq. (21)]

$$
M<\frac{R}{3}+\frac{2 Q^{2}}{3 R} \quad \text { for } \quad \frac{Q}{M} \leq \sqrt{\frac{9}{8}} .
$$

Taking cognizance of (11) and (22) one finds that, in the dimensionless regime $Q / M \leq \sqrt{9 / 8}$, the analytically derived upper bound (22) on the gravitational masses of stable spatially regular charged compact objects is stronger than the physically important bound (11). In particular, one finds that the newly derived upper bound (22) is stronger than (11) in the $R \geq Q$ regime. In addition, we recall that in the present paper we consider compact objects which are characterized by the dimensionless inequality $Q / M \leq \sqrt{9 / 8}$ [see (11)] which, taking cognizance of Eq. (21), corresponds to $R>\sqrt{2} Q$. One therefore concludes that, in the $Q / M \leq \sqrt{9 / 8}$ regime, the bound (22) for stable charged compact systems is stronger than the bound (11).

Finally, it is worth mentioning that a universal upper bound on the entropies of charged compact systems has been presented in 2, 26, 27]:

$$
S \leq \pi\left(2 M R-Q^{2}\right)
$$

Interestingly, substituting the newly derived upper bound (22) on the masses of physically realistic charged compact objects into (23), one can express the entropy upper bound in terms of the surface area $A=4 \pi R^{2}$ of the corresponding charged stable physical system [28]:

$$
S \leq \frac{A}{6}-\frac{\pi Q^{2}}{3}
$$

\section{ACKNOWLEDGMENTS}

This research is supported by the Carmel Science Foundation. I would like to thank Yael Oren, Arbel M. Ongo, Ayelet B. Lata, and Alona B. Tea for stimulating discussions.

[1] S. Chandrasekhar, The Mathematical Theory of Black Holes, (Oxford University Press, New York, 1983).

[2] We use natural units in which $G=c=\hbar=1$.

[3] H. A. Buchdahl, Phys. Rev. 116, 1027 (1959).

[4] H. Andréasson, Commun. Math. Phys. 288, 715 (2009).

[5] P. V. P. Cunha, E. Berti, and C. A. R. Herdeiro, Phys. Rev. Lett. 119, 251102 (2017).

[6] S. Hod, Phys. Lett. B 776, 1 (2018) arXiv:1710.00836.

[7] S. Hod, Phys. Lett. B 739, 383 (2014) arXiv:1412.3808.

[8] A null circular geodesic is considered to be stable (attractive) if massless perturbation fields tend to pile up on it.

[9] J. Keir, Class. Quant. Grav. 33, 135009 (2016).

[10] V. Cardoso, A. S. Miranda, E. Berti, H. Witek and V. T. Zanchin, Phys. Rev. D 79, 064016 (2009).

[11] S. Hod, Phys. Rev. D 84, 124030 (2011) arXiv:1112.3286]; S. Hod, Phys. Rev. D 84, 104024 (2011) arXiv:1201.0068; S. Hod, Phys Lett. B 727, 345 (2013) arXiv:1701.06587.

[12] This physically intriguing assertion is based on the fact that massless fields that propagate in the vicinity of unstable light rings tend to decay over time, whereas they tend to pile up and grow non-linearly around stable light rings [9].

[13] S. L. Shapiro and S. A. Teukolsky, Black Holes, White Dwarfs and Neutron Stars: The Physics of Compact Objects, 1st ed. (Wiley-Interscience, 1983).

[14] S. Hod, Phys. Rev. D 80, 064004 (2009) arXiv:0909.0314]; S. Hod, Phys. Lett. B 718, 1552 (2013) arXiv:1210.2486]; S. Hod, Phys. Lett. B 751, 177 (2015) arXiv:1707.06246]; S. Hod, Class. Quant. Grav. 33, 114001 (2016) arXiv:1705.08905. 
[15] Here $(t, r, \theta, \phi)$ are the Schwarzschild spacetime coordinates.

[16] A. E. Mayo and J. D. Bekenstein, Phys. Rev. D 54, 5059 (1996).

[17] We use the prime symbol' to denote a spatial derivative with respect to the radial coordinate $r$.

[18] H. Bondi, Mon. Not. Roy. Astr. Soc. 259, 365 (1992).

[19] We shall assume without loss of generality that $Q \geq 0$.

[20] As will be evident below, the external light ring (with $r_{\gamma}>R$ ) of the charged compact object exists in the dimensionless bounded regime (111) [see Eq. (20) below].

[21] Note that the conditions (12), which characterize the null circular geodesics of the spherically symmetric spacetime (2), correspond to $\dot{r}^{2}=\left(\dot{r}^{2}\right)^{\prime}=0[1,[10]$.

[22] We use the dot symbol $\cdot$ to denote a derivative with respect to an affine parameter.

[23] See [6] for the special case of horizonless compact objects which are characterized by degenerate light rings with $\mathcal{R}=\mathcal{R}^{\prime}=0$. As discussed in [6], these special matter configurations may possess an odd number of null circular geodesics.

[24] That is, the presence of a null circular geodesic in the exterior spacetime region with $\rho=p=0$ [see Eq. (18)].

[25] It is important to emphasize that it is a non-trivial task to prove this instability claim rigorously. Moreover, as emphasized in [9], even if a spacetime solution of the Einstein field equations is non-linearly unstable it is difficult to obtain estimates of the timescale which characterizes this instability. This implies that an unstable object could still be observed on finite timescales.

[26] J. D. Bekenstein and A. E. Mayo, Phys. Rev. D 61024022 (2000).

[27] S. Hod, Phys. Rev. D 61, 024023 (2000) arXiv:gr-qc/9903011.

[28] It is worth emphasizing again that in the present analysis we consider horizonless charged objects. On the other hand, black holes can violate the analytically derived upper bound (22), and as a consequence they can also violate the bound (24). 